MATHEMATICS OF COMPUTATION

Volume 68, Number 225, January 1999, Pages 73-89

S 0025-5718(99)01018-2

\title{
STABILITY OF RUNGE-KUTTA METHODS FOR ABSTRACT TIME-DEPENDENT PARABOLIC PROBLEMS: THE HÖLDER CASE
}

\author{
C. GONZÁLEZ AND C. PALENCIA
}

Abstract. We consider an abstract time-dependent, linear parabolic problem

$$
u^{\prime}(t)=A(t) u(t), \quad u\left(t_{0}\right)=u_{0},
$$

where $A(t): D \subset X \rightarrow X, t \in J$, is a family of sectorial operators in a Banach space $X$ with time-independent domain $D$. This problem is discretized in time by means of an $\mathrm{A}(\theta)$ strongly stable Runge-Kutta method, $0<\theta<\pi / 2$. We prove that the resulting discretization is stable, under the assumption

$$
\|(A(t)-A(s)) x\| \leq L|t-s|^{\alpha}(\|x\|+\|A(s) x\|), \quad x \in D, t, s \in J,
$$

where $L>0$ and $\alpha \in(0,1)$. Our results are applicable to the analysis of parabolic problems in the $L^{p}, p \neq 2$, norms.

\section{INTRODUCTION}

Let $X$ be a complex Banach space and let $J \subset \mathbf{R}$ be an interval. We consider a family of linear, densely defined operators $A(t): D \subset X \rightarrow X$, with domain $D(A(t))=D$ independent of $t \in J$. We are concerned with the the stability of discretizations in time, based on Runge-Kutta methods, of the initial value problem

$$
\left\{\begin{array}{l}
u^{\prime}(t)=A(t) u(t), \quad t \in J \\
u\left(t_{0}\right)=u_{0} \in D, \quad t_{0} \in J
\end{array}\right.
$$

For each angle $\theta \in(0, \pi / 2)$, we set

$$
S_{\theta}:=\{0\} \bigcup\{z \in \mathbf{C}: z \neq 0,|\arg (-z)| \leq \theta\} .
$$

Problem (1) is assumed to be parabolic in the sense that the operators are sectorial with constants independent of $t \in J$, i.e., we assume that the following condition holds.

H1. There exist $M \geq 1, \omega_{0} \in \mathbf{R}$ and $\theta \in(0, \pi / 2)$ such that, for a complex $z \notin$ $\omega_{0}+S_{\theta}$ and for $t \in J$, the resolvent $(z I-A(t))^{-1}: X \rightarrow X$ exists and the estimate

$$
\left\|(z I-A(t))^{-1}\right\| \leq \frac{M}{\left|z-\omega_{0}\right|}
$$

is satisfied.

Received by the editor June 5, 1996 and, in revised form September 4, 1996.

1991 Mathematics Subject Classification. Primary 65J10, 65M12, 65M15.

Key words and phrases. Parabolic problems, time-dependent, Hölder, Banach space, resolvents, sectorial, stability, Runge-Kutta.

(C)1999 American Mathematical Society 
The meaning of $\mathrm{H} 1$ is that the "frozen operator" problems

$$
\left\{\begin{array}{l}
u^{\prime}(t)=A\left(t^{*}\right) u(t), \quad t \in \mathbf{R}, \\
u\left(t_{0}\right)=u_{0} \in D
\end{array}\right.
$$

where $t^{*}$ ranges over $J$, are uniformly holomorphic. In fact, under H1 it is well known that for each angle $\varphi \in(0, \pi / 2-\theta)$ there exists $C=C(\varphi)>0$, independent of $t^{*} \in J$, such that $\left\|e^{\sigma A\left(t^{*}\right)}\right\| \leq C e^{\omega_{0}|\sigma|}$, for $-\sigma \in S_{\varphi}$.

For the applications we have in mind, including the future study of the stability of abstract quasilinear parabolic problems (see [11]), it is suitable to impose the relative Hölder variation of the coefficients $A(t), t \in J$. To be precise, we assume the following:

H2. There exist $L>0$ and $\alpha \in(0,1)$ such that

$$
\|(A(t)-A(s)) x\| \leq L|t-s|^{\alpha}(\|x\|+\|A(s) x\|), \quad x \in D, \quad t, s \in J .
$$

It is well known that $\mathrm{H} 1$ and $\mathrm{H} 2$ guarantee the existence and uniqueness of the solution of (1) (see e.g., [1, 2, 3, 13, 18, 19, 21]).

Problem (1) is discretized in time by means of a Runge-Kutta method defined by its Butcher array

$$
\left(\begin{array}{l|l}
\mathbf{c} & \mathcal{A} \\
\hline & \mathbf{b}^{T}
\end{array}\right)
$$

where $\mathbf{b}=\left[b_{1}, \ldots, b_{s}\right]^{T} \in \mathbf{R}^{s}, \mathbf{c}=\left[c_{1}, \ldots, c_{s}\right]^{T} \in \mathbf{R}^{s}$ and $\mathcal{A}=\left(a_{i j}\right)_{i, j=1}^{s} \in \mathbf{R}^{s \times s}$. We suppose that $0 \leq c_{i} \leq 1$, for $1 \leq i \leq s$. Let us recall that the stability function of the method is the rational function $r(z)=1+\mathbf{b}^{T}(\mathcal{I}-z \mathcal{A})^{-1} \mathbf{e}$, where $\mathcal{I} \in \mathbf{R}^{s \times s}$ stands for the identity matrix and $\mathbf{e}=[1, \ldots, 1]^{T} \in \mathbf{R}^{s}$. The method is $\mathrm{A}(\theta)$-stable, $0<\theta<\pi / 2$, when (i) the spectrum of the matrix $\mathcal{A}$ is contained in the complement of the sector $S_{\theta}$ and (ii) $|r(z)| \leq 1$, for $z \in S_{\theta}$. Notice that for A( $\left.\theta\right)$-stable methods, the matrix $\mathcal{A}$ is regular. Moreover, if the method also satisfies (iii) $\gamma:=|r(\infty)|<1$, then we say that the method is strongly $\mathrm{A}(\theta)$-stable. Hereafter, we only consider strongly $\mathrm{A}(\theta)$-stable methods. This excludes the Gaussian methods, among others. On the other hand, there is a wide range of methods lying within this class of strongly $\mathrm{A}(\theta)$-stable methods (see e.g., [12]).

Let $u: J \rightarrow X$ be the solution of problem (1). Let $t_{0}<t_{1}<\cdots<t_{N}$ be a finite sequence of time levels in $J$, with uniform spacing $h=t_{n+1}-t_{n}, 0 \leq n \leq N-1$. The application of the Runge-Kutta method given by (2) to problem (1) leads to the recurrence

$$
u_{n+1}=u_{n}+h \sum_{i=1}^{s} b_{i} A\left(t_{n}+c_{i} h\right) U_{n}^{i}, \quad 0 \leq n \leq N-1 .
$$

Here $u_{n}$ is the approximation to $u\left(t_{n}\right), 0 \leq n \leq N$, and the internal stages $U_{n}^{i} \in D$, $0 \leq n \leq N-1,1 \leq i \leq s$, are defined by the system of equations

$$
U_{n}^{i}=u_{n}+h \sum_{j=1}^{s} a_{i j} A\left(t_{n}+c_{j} h\right) U_{n}^{j}, \quad 1 \leq i \leq s .
$$

In Lemma 2.3 we prove that, assuming that the method is strongly $\mathrm{A}(\theta)$-stable, system (4) is uniquely solvable, for $h>0$ small enough, even for data $u_{n} \in X$ not lying in the domain $D$. In fact we show that, for $0 \leq n \leq N-1$, there exists a 
continuous, linear mapping $r\left(t_{n+1}, t_{n}\right): X \rightarrow X$ such that the recurrence (3) can be written in compact form as

$$
u_{n+1}=r\left(t_{n+1}, t_{n}\right) u_{n}, \quad 0 \leq n \leq N-1 .
$$

We also show that $r\left(t_{n+1}, t_{n}\right)$ maps $D$ onto $D$. Thus, the method makes sense for generalized as well as for genuine solutions. The main problem we address in the present paper is the stability of the procedure (5). Given a family $\left\{F_{j}\right\}_{j=n}^{m}$ of linear operators defined in a common space, we set

$$
\prod_{j=n}^{m} F_{j}=F_{m} \cdot F_{m-1} \cdots F_{n} .
$$

The stability of the method demands the boundedness, in independence of $h>0$ small enough, of the compositions $\prod_{j=n}^{m} r\left(t_{j+1}, t_{j}\right)$ as bounded operators in $X$. Let us point out that in the present paper we address not only the question of the stability, but also the question of the so-called strong stability (see below) of the method. The strong stability result turns out to be basic for the study of the stability of the discretizations of quasilinear problems in [11]. We pay attention to the size of the stability constants. This point is very important for the study of quasilinear problems, as well as for the study of the asymptotic behavior of the numerical solution.

For the proof of our results we require intermediate spaces between $D$ and $X$. The domain $D$ is assumed to be endowed with the graph norm $\|\cdot\|_{1}$ corresponding to any $A\left(t^{*}\right), t^{*} \in J$, i.e.,

$$
\|x\|_{1}:=\|x\|+\left\|A\left(t^{*}\right) x\right\|, \quad x \in D,
$$

where $t^{*} \in J$ has been fixed. After H2, any pair of such norms, corresponding to different choices of $t^{*} \in J$, are mutually equivalent (see below). The space $D$ is Banach, since the operators $A\left(t^{*}\right), t^{*} \in J$, are closed. We set $X_{0}=X, X_{1}=D$ and, for $0 \leq \eta \leq 1$, we denote by $X_{\eta}=\left[X_{0}, X_{1}\right]_{\eta}$ the Calderón interpolation space of order $\eta$ between $X_{0}$ and $X_{1}$ (see e.g., [5, 22]). Only the basic interpolation properties are used in our analysis, so that the reader does not need a deep knowledge of interpolation theory. Let us point out that the interpolation spaces obtained by the real method could be used instead (see e.g., [5, 22]). However, the apparently simpler choice $X_{\eta}=D\left(\left(\omega_{0} I-A(t)\right)^{\eta}\right)$, the domain of the fractional power, is troublesome since, due to the lack of validity of Heinz's theorem, such a domain may depend on $t \in J$. The operator norm of a bounded linear operator $F: X_{\mu} \rightarrow X_{\nu}$, where $\mu, \nu \in[0,1]$, is denoted by $\|F\|_{\mu \rightarrow \nu}$. We set $\tilde{\omega}_{0}=\omega_{0} / 2$, for $\omega_{0} \leq 0$, and $\tilde{\omega}_{0}=3 \omega_{0} / 2$, for $\omega_{0}>0$. With this notation we can state the following theorem, which provides the main contribution of the present paper.

Theorem 1.1. Assume that the parabolic problem (1) fulfills hypotheses $H 1$ and H2, for some $M \geq 1, \omega_{0} \in \mathbf{R}, \theta \in(0, \pi / 2), L \geq 0$ and $\alpha \in(0,1)$, and assume that the Runge-Kutta method given by (2) is strongly $A(\theta)$-stable. Then there exist constants $K>0$ and $\Omega>0$, that are independent of $L$, and there exists $\bar{h}>0$ such that for any arbitrary finite sequence of time levels $t_{j}, 0 \leq j \leq N$, in $J$ with constant step-size $0<h \leq \bar{h}$ the stage equations (4) are uniquely solvable in $X$ and 
the following stability estimates hold:

$$
\begin{aligned}
& \left\|\prod_{j=0}^{N-1} r\left(t_{j+1}, t_{j}\right)-\gamma^{N} I\right\|_{0 \rightarrow \mu} \leq K T^{-\mu} e^{\left(\tilde{\omega}_{0}+\Omega L^{1 / \alpha}\right) T}\left(1+B L T^{\alpha}\right)^{5}, \\
& 0 \leq \mu<1, \\
& \left\|\prod_{j=0}^{N-1} r\left(t_{j+1}, t_{j}\right)-\gamma^{N} I\right\|_{\nu \rightarrow 1} \leq K T^{\nu-1} e^{\left(\tilde{\omega}_{0}+\Omega L^{1 / \alpha}\right) T}\left(1+B L T^{\alpha}\right)^{5}, \\
& \left\|\prod_{j=0}^{N-1} r\left(t_{j+1}, t_{j}\right)-\gamma^{N} I\right\|_{0 \rightarrow 1} \leq K T^{-1} e^{2\left(\tilde{\omega}_{0}+\Omega L^{1 / \alpha}\right) T}\left(1+B L T^{\alpha}\right)^{10},
\end{aligned}
$$

where $r\left(t_{j+1}, t_{j}\right), 0 \leq j \leq N-1$, are the operators defined in (5) and $T=t_{N}-t_{0}$. In (7), respectively in (8), $B>0$ depends on $\gamma$ and $\mu$, respectively on $\gamma$ and $\nu$.

Notice that for either $\mu=0$ in (7) or $\nu=1$ in (8) we can dispense with the term $\gamma^{N}$. Therefore, Theorem 1.1 yields the stability of the Runge-Kutta method in either $X_{0}$ or $X_{1}$. Furthermore, by interpolation, we deduce that the Runge-Kutta method is stable in $X_{\mu}$, for $0 \leq \mu \leq 1$, and that we have the bound

$$
\left\|\prod_{j=0}^{N-1} r\left(t_{j+1}, t_{j}\right)\right\|_{\mu \rightarrow \mu} \leq K e^{\left(\tilde{\omega}_{0}+\Omega L^{1 / \alpha}\right) T}\left(1+B L T^{\alpha}\right)^{5} .
$$

The way $L$ enters in the estimates in Theorem 1.1 is crucial for the applications in [11]. Moreover, we see that in case of asymptotic stability, i.e., when $\omega_{0}<0$, and for small enough $L$, Theorem 1.1 yields estimates that are uniform, even with an exponential damping, in $t \in J$. This is an important remark from the qualitative point of view.

The estimate (9) can be viewed as the discrete counterpart of the analyticity of the continuous problem. It shows that, except for the term $\gamma^{n} u_{0}$, the numerical approximations $u_{n}, 1 \leq n \leq N$, in (5) are smooth (in the sense that they belong to $X_{1}$ ) even for non-smooth initial data $u_{0} \in X$. When $\gamma=0,(9)$ yields the so-called strong stability of the method. At first glance, it may seem natural to first prove (9) and then obtain (7) and (8) by interpolation. However, for the proof of the previous estimates we need some sort of Gronwall's lemma (see Lemma 2.1) for weakly singular convolution kernels. This lemma cannot be applied directly to the proof of (9) because a non-integrable singularity appears. Therefore, in the proof of Theorem 1.1, (9) is obtained as a consequence of (7) and (8).

For the backward Euler method, stability was studied in $[9,20]$. In [4] similar stability results for higher order methods are stated. However, the strong stability concept considered in [4] differs from ours, because in [4], in the definition of the intermediate spaces $X_{\eta}, 0 \leq \eta \leq 1$, the graph norm $\widehat{A(t)} \eta^{\eta}$ of the discrete generator $\widehat{A(t)}:=(I-r(h A(t))) / h, t \in J$, is used instead of our choice. On the other hand, conclusions related to ours, but in the context of Hilbert spaces and GelfandLions triplets, were obtained in [15]. Our general Banach spaces set-up cannot take advantage on the main ideas used in [15]. Finally, in [10] we studied the stability for time dependent problems (1), but there we assumed that the relative total variaton of the operators $A(t), t \in J$, was bounded. In [10] methods with $|r(\infty)|=1$ can be 
considered. Moreover, in [10] we were able to give a precise account of the size of the stability constants obtained. Under the hypothesis $\mathrm{H} 2$, the perturbative argument of [10] cannot be used any longer.

The applications of Theorem 1.1 include the semidiscretizations in time of classical parabolic problems in the $L^{p}, 1 \leq p \leq+\infty$, spaces. The reader is referred to [10, Section 5], but taking into account that now, after Theorem 1.1, the coefficients are allowed to be Hölder continuous in time. The main limitation, as in [10], is that the domain of the operators must be independent of $t \in J$. This may exclude Neumann boundary conditions. Finally, as we have already mentioned, Theorem 1.1 is also basic for the study of the semidiscretizaton in time of abstract quasilinear parabolic problems (see [11]). Let us point out that for this study it is important to reflect the dependence of the bounds on $L$ and $\alpha$, as in Theorem 1.1.

In Section 2 we present some auxiliary lemmas needed for the proof of Theorem 1.1, including the Gronwall-type lemma. Section 3 is devoted to the proof of Theorem 1.1.

\section{SOME AUXILIARY LEMMAS}

In this section we present some lemmas that are necessary for the proof of Theorem 1.1, we maintain the notation and hypotheses of this theorem. We assume that we have fixed a uniformily spaced sequence $t_{j}, 0 \leq j \leq N$, in $J$, with step $h>0$.

The first lemma provides a version of Gronwall's lemma with a weakly singular kernel. It is noteworthy that this lemma, in spite of its simple appearence, cannot be obtained directly by comparison with its continuous counterpart. Another version of a similar lemma can be found in [14]. We prefer our statement to that in [14] since it accounts for the dependence with respect to the parameters involved. A non-standard term is also included. This term will allow us to consider methods with $r(\infty) \neq 0$.

Lemma 2.1. Let $h>0, N \geq 1$ integer and set $t_{j}=j h, 0 \leq j \leq N$. Let $\xi_{j} \geq 0$, $0 \leq j \leq N$, be a finite sequence of real numbers with $\xi_{0}=0$. Assume that there exist $\alpha \in(0,1), \eta, \gamma \in[0,1)$ and $C_{1}, C_{2}, C_{3} \geq 0$ such that $h \leq \bar{h}:=\left((1-\gamma)^{2} /\left(4 C_{2}\right)\right)^{1 / \alpha}$ and that, for $1 \leq m \leq N$, we have

$$
\begin{aligned}
\xi_{m} \leq C_{1} t_{m}^{-\eta} & +C_{2} \sum_{j=1}^{m-1}\left(h t_{m-j}^{\alpha-1}+t_{m-j}^{\alpha} \gamma^{m-j-1}\right) \xi_{j} \\
& +C_{3} \sum_{j=1}^{m-1}\left(h^{1-\eta} t_{m-j}^{\alpha-1} \gamma^{j}+h^{-\eta} \gamma^{m-1} t_{m-j}^{\alpha}\right) .
\end{aligned}
$$

Then there exists a constant $B \geq 0$, depending only on $\eta$ and $\gamma$, such that the estimate

$$
\xi_{m} \leq 2 e^{\omega t_{m}}\left(C_{1}+B C_{3} t_{m}^{\alpha}\right)\left(1+B C_{2} t_{m}^{\alpha}\right) t_{m}^{-\eta}, \quad 1 \leq m \leq N
$$

holds with

$$
\omega=\left(4 C_{2} \Gamma(\alpha)\right)^{1 / \alpha}
$$


Proof. We begin by proving that there exists $B_{1}>0$, depending only on $\eta$ and $\gamma$, such that

$$
\sum_{j=1}^{m-1}\left(h^{1-\eta} t_{m-j}^{\alpha-1} \gamma^{j}+h^{-\eta} \gamma^{m-1} t_{m-j}^{\alpha}\right) \leq B_{1} t_{m}^{\alpha-\eta}, \quad 1 \leq m \leq N .
$$

We can assume that $\gamma>0$. Fix $1 \leq m \leq N$ and let $M$ be the integer part of $\left(m^{1-\eta}\right) / 2$. We have that

$$
\begin{aligned}
h^{1-\eta} \sum_{j=1}^{m-1} t_{m-j}^{\alpha-1} \gamma^{j} & \leq h^{1-\eta} \sum_{j=1}^{M} t_{m-j}^{\alpha-1}+h^{1-\eta} \sum_{j=M+1}^{m-1} t_{m-j}^{\alpha-1} \gamma^{j} \\
& \leq h^{1-\eta} M t_{m-M}^{\alpha-1}+h^{\alpha-\eta} m \gamma^{M+1} \\
& \leq \frac{M m^{\eta}}{m-M} t_{m}^{\alpha-\eta}+m^{2} \gamma^{M+1} t_{m}^{\alpha-\eta} \\
& \leq B^{\prime} t_{m}^{\alpha-\eta}
\end{aligned}
$$

where $B^{\prime}:=1+\sup _{x \geq 0} x^{2} \gamma^{(1 / 2) x^{1-\eta}}$. Moreover, we have

$$
h^{-\eta} \sum_{j=1}^{m-1} \gamma^{m-1} t_{m-j}^{\alpha} \leq t_{m}^{\alpha-\eta} m^{1+\eta} \gamma^{m-1} \leq B^{\prime \prime} t_{m}^{\alpha-\eta},
$$

where $B^{\prime \prime}:=\sup _{x \geq 1} x^{1+\eta} \gamma^{x-1}$. Therefore, (12) holds with $B_{1}=B^{\prime}+B^{\prime \prime}$.

Now, after (12), the proof of the lemma can be restricted to the case $C_{3}=0$. Furthermore, with no loss of generality, we can assume that $C_{1}=1$. It is also clear that it is sufficient to prove (11) for $m=N$, because then the same result could be applied to a smaller value of $1 \leq m \leq N$.

Let $\mathbf{g}$ and $\mathbf{t}_{\sigma}, \sigma>0$, be the sequences defined by $\mathbf{g}(j)=t_{j}^{\alpha} \gamma^{j-1}$ and $\mathbf{t}_{\sigma}(j)=t_{j}^{-\sigma}$, for $1 \leq j \leq N$, and by $\mathbf{g}(j)=\mathbf{t}_{\sigma}(j)=0$, for the remaining values of $j \geq 0$ integer. Furthermore, let $\mathbf{x}=\left\{x_{j}\right\}_{j=0}^{+\infty}$ be the sequence defined by the convolution equation

$$
\mathbf{x}=\mathbf{t}_{\eta}+C_{2}\left(h \mathbf{t}_{1-\alpha}+\mathbf{g}\right) * \mathbf{x},
$$

where $*$ stands for the discrete convolution of sequences. It is obvious that we have $\xi_{j} \leq x_{j}$, for $1 \leq j \leq N$. Therefore the lemma is reduced to prove that, for $j=N$, inequality (11) holds with $x_{N}$ instead of $\xi_{N}$.

For each given sequence $\mathbf{u}=\left\{u_{j}\right\}_{j=0}^{+\infty}$ of complex numbers, we set $\tilde{\mathbf{u}}(z)=$ $\sum_{j=0}^{+\infty} u_{j} z^{j}$, i.e., $\tilde{\mathbf{u}}$ stands for the generating function of $\mathbf{u}$. As is well known, in terms of the generating functions, equation (13) becomes

$$
\tilde{\mathbf{x}}(z)=\frac{\tilde{\mathbf{t}}_{\eta}(z)}{1-C_{2}\left(h \tilde{\mathbf{t}}_{1-\alpha}(z)+\tilde{\mathbf{g}}(z)\right)} .
$$

Let $\mathbf{r}=\left\{r_{j}\right\}_{j=0}^{+\infty}$ and $\mathbf{s}=\left\{s_{j}\right\}_{j=0}^{+\infty}$ be the sequences whose generating functions are $\tilde{\mathbf{r}}(z)=\left(1-C_{2}\left(h \tilde{\mathbf{t}}_{1-\alpha}(z)+\tilde{\mathbf{g}}(z)\right)\right)^{-1}$ and $\tilde{\mathbf{s}}(z)=\tilde{\mathbf{r}}(z)^{2}$, respectively. Now we transform (14) by taking the derivatives and multiplying by $z$ (recall that this process corresponds to taking the generating function of the original sequence multiplied componentwise by the sequence $\left.\{j\}_{j=0}^{+\infty}\right)$. This leads to

$$
z \tilde{\mathbf{x}}^{\prime}(z)=z \tilde{\mathbf{t}}_{\eta}^{\prime}(z) \tilde{\mathbf{r}}(z)+C_{2} z \tilde{\mathbf{t}}_{\eta}(z)\left(h \tilde{\mathbf{t}}_{1-\alpha}^{\prime}(z)+\tilde{\mathbf{g}}^{\prime}(z)\right) \tilde{\mathbf{s}}(z),
$$


that after inverting leads to

$$
N x_{N}=\sum_{j=1}^{N} r_{N-j} j t_{j}^{-\eta}+C_{2} \sum_{j=1}^{N} s_{N-j} \sum_{l=1}^{j-1} t_{j-l}^{-\eta}\left(h t_{l}^{\alpha-1}+t_{l}^{\alpha} \gamma^{l-1}\right) .
$$

Let us assume for the moment the validity of the estimates

$$
\sum_{j=0}^{N}\left|r_{j}\right| \leq 2 e^{\omega t_{N}}, \quad \sum_{j=0}^{N}\left|s_{j}\right| \leq 4 e^{\omega t_{N}}
$$

which we prove later. For $1 \leq j \leq N$, we have that

$$
j t_{j}^{-\eta} \leq N t_{N}^{-\eta}
$$

and also, since $B(1+\alpha, 1-\eta) \leq 2 / \Gamma(1-\eta)$, that

$$
\begin{aligned}
\sum_{l=1}^{j-1} t_{j-l}^{-\eta} l\left(h t_{l}^{\alpha-1}+t_{l}^{\alpha} \gamma^{l-1}\right) & \leq\left(1+\gamma^{*}\right) \sum_{l=1}^{j-1} t_{j-l}^{-\eta} t_{l}^{\alpha} \\
& \leq\left(1+\gamma^{*}\right) h^{-1} \int_{0}^{t_{j}}\left(t_{j}-\tau\right)^{-\eta} \tau^{\alpha} d \tau \\
& =\left(1+\gamma^{*}\right) j B(1+\alpha, 1-\eta) t_{j}^{\alpha-\eta} \\
& \leq 2\left(1+\gamma^{*}\right) \Gamma(1-\eta)^{-1} N t_{N}^{\alpha-\eta}
\end{aligned}
$$

where $\gamma^{*}=\sup _{x \geq 1} x \gamma^{x-1}$. By using the previous estimates in (15), it is straightforward to conclude that

$$
\begin{aligned}
N x_{N} & \leq\left(\sum_{j=0}^{N}\left|r_{j}\right|\right) N t_{N}^{-\eta}+2 C_{2}\left(\sum_{j=0}^{N}\left|s_{j}\right|\right) N t_{N}^{\alpha-\eta}\left(1+\gamma^{*}\right) \Gamma(1-\eta)^{-1} \\
& \leq 2 N t_{N}^{-\eta} e^{\omega t_{N}}\left(1+4 C_{2} \Gamma(1-\eta)^{-1}\left(1+\gamma^{*}\right) t_{N}^{\alpha}\right),
\end{aligned}
$$

and, dividing by $N$, we get the desired bound for $x_{N}$ with

$$
B=\max \left\{B_{1}, 4 \Gamma(1-\eta)^{-1}\left(1+\gamma^{*}\right)\right\}
$$

It remains to prove (16). Notice that $\tilde{\mathbf{t}}_{1-\alpha}(0)=\tilde{\mathbf{g}}(0)=0$. Thus, for $z \in \mathbf{C}$ with small enough $|z|$, we have that

$$
\tilde{\mathbf{r}}(z)=\sum_{k=0}^{+\infty}\left(C_{2}\left(h \tilde{\mathbf{t}}_{1-\alpha}(z)+\tilde{\mathbf{g}}(z)\right)\right)^{k}
$$


hence $r_{j} \geq 0$, for all $j \geq 0$, since all the coefficients of $h \tilde{\mathbf{t}}_{1-\alpha}(z)+\tilde{\mathbf{g}}(z)$ are nonnegative. Moreover, recalling the definition of $\omega$ and $\bar{h}$, it is clear that

$$
\begin{aligned}
C_{2}\left(h \tilde{\mathbf{t}}_{1-\alpha}\left(e^{-h \omega}\right)+\tilde{\mathbf{g}}\left(e^{-h \omega}\right)\right) & =C_{2} h \sum_{j=1}^{N}\left(t_{j}^{\alpha-1}+h^{-1} t_{j}^{\alpha} \gamma^{j-1}\right) e^{-\omega t_{j}} \\
& \leq C_{2} \int_{0}^{t_{N}} \frac{e^{-\omega u}}{u^{1-\alpha}} d u+C_{2} \sum_{j=1}^{N} t_{j}^{\alpha} \gamma^{j-1} \\
& \leq C_{2} \omega^{-\alpha} \int_{0}^{\omega t_{N}} e^{-v} v^{\alpha-1} d v+C_{2} h^{\alpha} \sum_{j=1}^{N} j \gamma^{j-1} \\
& \leq C_{2} \Gamma(\alpha) \omega^{-\alpha}+C_{2} h^{\alpha}(1-\gamma)^{-2} \\
& \leq 1 / 4+1 / 4=1 / 2 .
\end{aligned}
$$

Therefore, we have

$$
\begin{aligned}
\sum_{j=0}^{N}\left|r_{j}\right| & =\sum_{j=1}^{N} r_{j} \leq e^{\omega t_{N}} \sum_{j=1}^{N} e^{-\omega t_{j}} r_{j} \\
& =e^{\omega t_{N}} \tilde{\mathbf{r}}\left(e^{-\omega h}\right) \\
& =e^{\omega t_{N}}\left(1-C_{2}\left(h \tilde{\mathbf{t}}_{1-\alpha}\left(e^{-h \omega}\right)+\tilde{\mathbf{g}}\left(e^{-h \omega}\right)\right)\right)^{-1} \\
& \leq 2 e^{\omega t_{N}}
\end{aligned}
$$

In the same way we see that

$$
\sum_{n=0}^{N}\left|s_{j}\right| \leq e^{\omega t_{N}}\left(1-C_{2}\left(h \tilde{\mathbf{t}}_{1-\alpha}\left(e^{-h \omega}\right)+\tilde{\mathbf{g}}\left(e^{-h \omega}\right)\right)\right)^{-2} \leq 4 e^{\omega t_{N}} .
$$

Hereafter, the letter $K$ possibly with a subindex denotes positive constants that depend only on $M, \theta, \omega_{0}$ and the Runge-Kutta method. Of course, the $K$ 's may take different values at different places.

Lemma 2.2. There exist $K>0$ and $\bar{h}>0$, depending on $M, \theta, \omega_{0}$ and the RungeKutta method such that for all $t \in J, n \geq 1$ integer and $0<h<\bar{h}$, the following estimate holds:

$$
\left\|A(t)\left(r^{n}(h A(t))-\gamma^{n} I\right)\right\|_{0 \rightarrow 0} \leq \frac{K e^{\tilde{\omega}_{0} n h}}{n h} .
$$

Proof. The proof of this lemma is based on the Cauchy formula and it follows closely the proof of the main theorem in [17].

Assume first that $\omega_{0}=0$. Select $h>0, t \in J$ and set $A=h A(t)$. By using the Neumann series (see, e.g., in [8, Lemma 4.2.1]), it is easy to see that there exist $M^{*} \geq M$ and $0<\theta^{*}<\theta$, depending only on $M$ and $\theta$, such that $A$ satisfies H1 with respect $M^{*}, \theta^{*}$ and $\omega_{0}=0$. Then, because of the maximum principle, we have $|r(z)|<1$, for $z \neq 0, z \in S_{\theta^{*}}$. Since $\gamma=|r(\infty)|<1$, it is not hard to conclude that there exist $c>0,0<\bar{\gamma}<1$ and $R>0$, depending only on $M, \theta$ and $r(z)$, such that

$$
|r(z)| \leq \begin{cases}e^{c|z|}, & \text { if } \quad|z| \leq R, \quad z \notin S_{\theta^{*}} \\ e^{-c|z|}, & \text { if } \quad|z| \leq R, \quad z \in S_{\theta^{*}} \\ \bar{\gamma}, & \text { if } \quad|z| \geq R, \quad z \in S_{\theta^{*}}\end{cases}
$$


Select two radii $0<R_{0}<R<R_{\infty}$ in such a way that all the poles of $r(z)$ lay in the annulus $R_{0}<|z|<R_{\infty}$. For $n \geq 1$ integer, let $\Gamma_{n}$ be the negative boundary of the intersection of the annulus $R_{0} / n \leq|z| \leq R_{\infty}$ with the complement of the sector $S_{\theta^{*}}$. Following [17], we write

$$
A\left(r^{n}(A)-\gamma^{n} I\right)=\frac{1}{2 \pi i} \int_{\Gamma_{n}} z\left(r(z)^{n}-\gamma^{n}\right)(z I-A)^{-1} d z .
$$

In order to estimate this integral, we first partition $\Gamma_{n}$ as

$$
\Gamma_{n}=\Gamma_{n, 0} \bigcup \Gamma_{\infty} \bigcup L_{n} \bigcup L_{\infty}
$$

where $\Gamma_{n, 0}$ (respectively $\Gamma_{\infty}$ ) is the part of $\Gamma_{n}$ on the circle $|z|=R_{0} / n$ (respectively $|z|=R_{\infty}$ ) and $L_{n}$ (respectively $L_{\infty}$ ) is the part of $\Gamma_{n}$ on the boundary of the sector $S_{\theta^{*}}$ lying in the disk $|z| \leq R$ (respectively in the region $|z| \geq R$ ). After the representation (18), we have

$$
A\left(r^{n}(A)-\gamma^{n} I\right)=I_{1, n}+I_{2, n}+I_{3, n}+I_{4, n},
$$

where $I_{1, n}, I_{2, n}, I_{3, n}$ and $I_{4, n}$ stand for the contributions to the integral due to $\Gamma_{n, 0}, \Gamma_{\infty}, L_{n}$, and $L_{\infty}$, respectively. By taking into account that $\mathrm{H} 1$ holds for $A$, with $M^{*}, \theta^{*}$ and $\omega_{0}=0$, and by (17), it is straightforward to see that there exists $K_{0}>0$ such that

$$
\begin{aligned}
\left\|I_{1, n}\right\|_{0 \rightarrow 0} \leq M^{*}\left(R_{0} / n\right)\left(e^{c R_{0}}+\gamma^{n}\right) \leq K_{0} / n, \\
\left\|I_{2, n}\right\|_{0 \rightarrow 0} \leq M^{*} R_{\infty}\left(\bar{\gamma}^{n}+\gamma^{n}\right) \leq K_{0} / n, \\
\left\|I_{3, n}\right\|_{0 \rightarrow 0} \leq\left(M^{*} / \pi\right) \int_{0}^{R}\left(e^{-n c s}+\gamma^{n}\right) d s \leq K_{0} / n, \\
\left\|I_{4, n}\right\|_{0 \rightarrow 0} \leq\left(M^{*} R_{\infty} / \pi\right)\left(\bar{\gamma}^{n}+\gamma^{n}\right) \leq K_{0} / n .
\end{aligned}
$$

These estimates in (18) yield

$$
\left\|A(t)\left(r^{n}(h A(t))-\gamma^{n} I\right)\right\|_{0 \rightarrow 0}=h^{-1}\left\|A\left(r^{n}(A)-\gamma^{n} I\right)\right\|_{0 \rightarrow 0} \leq 4 K_{0} /(n h) .
$$

Therefore, for $\omega_{0}=0,(17)$ holds with $\bar{h}=+\infty$.

Assume that $\omega_{0} \neq 0$. If $\omega_{0}>0$, let $\bar{h}>0$ be such that all the poles of $r_{h}(z)$ lay outside the sector $S_{\theta}$. If $\omega_{0}<0$, let $\bar{h}=|\ln \bar{\gamma}| /\left|\omega_{0}\right|$. For $0<h<\bar{h}$, we set $r_{h}(z)=r\left(z+h \omega_{0}\right)$. Fix $0<h<\bar{h}, t \in J$ and set $A=h\left(A(t)-\omega_{0} I\right)$. Notice that $A$ satisfies H1, but with $\omega_{0}=0$. Now, for $n \geq 1$, we have

$$
A\left(r_{h}^{n}(A)-\gamma^{n} I\right)=\frac{1}{2 \pi i} \int_{\Gamma_{n}} z\left(r_{h}^{n}(z)-\gamma^{n}\right)(z I-A)^{-1} d z .
$$

It is not hard to see, due to our choice of $\bar{h}$, that there exists $K_{1}>0, R>0$, $\bar{\gamma} \in(0,1)$ and $c>0$, such that $r_{h}^{n}(z)$ satisfies the following estimates, that are similar to the ones satisfied by $r(z)$ in (17):

$$
\left|r_{h}(z)^{n}\right| \leq\left\{\begin{array}{lll}
K_{1} e^{c n|z|} e^{\omega_{0} n h}, & \text { if } \quad|z| \leq R, \quad z \notin S_{\theta^{*}}, \\
K_{1} e^{-c n|z|} e^{\omega_{0} n h}, & \text { if } \quad|z| \leq R, \quad z \in S_{\theta^{*}}, \\
K_{1} \bar{\gamma}^{n} e^{\omega_{0} n h}, & \text { if } \quad|z| \geq R, \quad z \in S_{\theta^{*}} .
\end{array}\right.
$$

Then, by partitioning $\Gamma_{n}$ as we did in the previous case, we can estimate the integral in (19) and show that there exists $K_{2}>0$ such that

$$
\left\|A\left(r_{h}^{n}(A)-\gamma^{n} I\right)\right\|_{0 \rightarrow 0} \leq K_{2} e^{\omega_{0} n h} / n .
$$


On the other hand, it is known (see $[6,7,16,17]$ ) that there exists $K_{3}>0$ such that

$$
\left\|r^{n}(h A(t))\right\|_{0 \rightarrow 0} \leq K_{3} e^{\omega_{0} n h} .
$$

Therefore,

$$
\begin{aligned}
\left\|A(t)\left(r^{n}(h A(t))-\gamma^{n} I\right)\right\|_{0 \rightarrow 0} \leq & \left|\omega_{0}\right|\left(\left\|r^{n}(h A(t))\right\|_{0 \rightarrow 0}+\gamma^{n}\right) \\
& +h^{-1}\left\|A\left(r_{h}^{n}(A)-\gamma^{n} I\right)\right\|_{0 \rightarrow 0} \\
\leq & \left|\omega_{0}\right|\left(K_{3} e^{\omega_{0} n h}+e^{\omega_{0} n h}\right)+K_{2} e^{\omega_{0} n h} /(n h),
\end{aligned}
$$

and the lemma is proved, since it is clear that $e^{\omega_{0} n h} \leq K_{4} e^{\tilde{\omega}_{0} n h} /(n h)$, for some $K_{4}>0$.

For each $t \in J$, we consider the norm $\|\cdot\|_{1}^{t}$ in $X_{1}=D$ defined by $\|x\|_{1}^{t}=$ $\|x\|+\|A(t) x\|$, for $x \in X_{1}$. Because of H2, we have

$$
\left(1+L|t-s|^{\alpha}\right)^{-1}\|x\|_{1}^{t} \leq\|x\|_{1}^{s} \leq\left(1+L|t-s|^{\alpha}\right)\|x\|_{1}^{t}, \quad x \in X_{1}, \quad t, s \in J .
$$

For $\mu \in(0,1),\|\cdot\|_{\mu}^{t}$ stands for the norm in the intermediate space $X_{\mu}=\left[X, X_{1}\right]_{\mu}$ obtained by means of the complex interpolation method, between $(X,\|\cdot\|)$ and $\left(X_{1},\|\cdot\|_{1}^{t}\right)$. The product space $X_{\mu}^{k}, k \geq 1$ integer and $0 \leq \mu \leq 1$, is endowed with the maximum norm component-wise. The norm in $X_{\mu}^{k}$ is also denoted by $\|\cdot\|_{\mu}^{t}$. Given $l, k \geq 1$ integers and $\nu \in[0,1]$, the operator norm corresponding to a bounded operator $F:\left(X_{\mu}^{l},\|\cdot\|_{\mu}^{s}\right) \rightarrow\left(X_{\nu}^{k},\|\cdot\|_{\nu}^{t}\right)$ is denoted by $\|F\|_{\mu \rightarrow \nu}^{s \rightarrow t}, s, t \in J$. For $\mu=\nu=0$, we simply set $\|F\|_{0 \rightarrow 0}$ instead of $\|F\|_{0 \rightarrow 0}^{s \rightarrow t}$. At first glance, it appears more natural to fix $t^{*} \in J$ and consider always the norm $\|\cdot\|_{1}^{t^{*}}$ in $X_{1}$. In this way, we could have fixed norms in the product spaces $X_{\mu}^{l}$ and $X_{\nu}^{k}$ and, consequently, we could avoid the cumbersome notation above for the norm of the operators $F: X_{\mu}^{l} \rightarrow X_{\nu}^{k}$. However, with our technique, such a choice of the norm in $X_{1}$ leads to an extra factor in the estimates in Theorem 1.1. This extra factor turns out to be of the form $e^{c T}$, where $c>0$ is independent of $L$, and, with this factor, we could not prove any result on asymptotic stability.

A matrix $\mathcal{M} \in \mathbf{C}^{k \times l}$ is identified with the operator $\mathcal{M} \otimes I: X_{\mu}^{l} \rightarrow X_{\mu}^{k}$. For $t \in J$ such that $t+h \in J$, we set $B(t), B_{0}(t): D^{s} \subset X^{s} \rightarrow X^{s}$ the operators defined by $B(t)=\operatorname{diag}\left(A\left(t+c_{1} h\right), \ldots, A\left(t+c_{s} h\right)\right)$ and by $B_{0}(t)=\operatorname{diag}(A(t), \ldots, A(t))$, respectively.

The solvability of the equations of the stages (4) is a direct consequence of the following lemma.

Lemma 2.3. There exists $K>0$ and there exists $\bar{h}>0$, with $\bar{h}$ depending on $M, \theta$, the Runge-Kutta method, $L$ and $\alpha$, such that, for $t \in J$ and $0<h<\bar{h}$ with $t+h \in J$, the operators $(\mathcal{I}-h \mathcal{A} B(t)),\left(\mathcal{I}-h \mathcal{A} B_{0}(t)\right): D^{s} \subset X^{s} \rightarrow X^{s}$ are boundedly invertible with

$$
\left\|(\mathcal{I}-h \mathcal{A} B(t))^{-1}\right\|_{0 \rightarrow 0} \leq K, \quad\left\|\left(\mathcal{I}-h \mathcal{A} B_{0}(t)\right)^{-1}\right\|_{0 \rightarrow 0} \leq K .
$$

Proof. As shown in the proof of Theorem 4.1 in [10], there exist constants $K_{1}>0$ and $h_{0}>0$, with $h_{0}$ depending only on $M, \theta \omega_{0}$ and $\mathcal{A}$, such that, for $0<h<h_{0}$, the inverse $\left(\mathcal{I}-h \mathcal{A} B_{0}(t)\right)^{-1}$ exists as a bounded operator in $X^{s}$ and

$$
\left\|\left(\mathcal{I}-h \mathcal{A} B_{0}(t)\right)^{-1}\right\|_{0 \rightarrow 0} \leq K_{1}, \quad\left\|h B_{0}(t)\left(\mathcal{I}-h \mathcal{A} B_{0}(t)\right)^{-1}\right\|_{0 \rightarrow 0} \leq a\left(1+K_{1}\right),
$$


where $a=\left\|\mathcal{A}^{-1}\right\|_{0 \rightarrow 0}$. Fix $0<h<h_{0}$. Then it makes sense to define the operator $\Delta(h): X^{s} \rightarrow X^{s}$ by $\Delta(h)=h \mathcal{A}\left(B(t)-B_{0}(t)\right)\left(\mathcal{I}-h \mathcal{A} B_{0}(t)\right)^{-1}$. By hypothesis H1, we have

$$
\|\Delta(h)\|_{0 \rightarrow 0} \leq\|\mathcal{A}\|_{0 \rightarrow 0} L h^{\alpha}\left\|h B_{0}(t)\left(\mathcal{I}-h \mathcal{A} B_{0}(t)\right)^{-1}\right\|_{0 \rightarrow 0} \leq K_{0} L h^{\alpha} .
$$

By writing

$$
\mathcal{I}-h \mathcal{A} B(t)=(\mathcal{I}-\Delta(h))\left(\mathcal{I}-h \mathcal{A} B_{0}(t)\right)
$$

we see that, for $h<\bar{h}:=\min \left\{h_{0},\left(2 K_{0} L\right)^{-1 / \alpha}\right\}$, the inverse $(\mathcal{I}-h \mathcal{A} B(t))^{-1}$ exists and

$$
\left\|(\mathcal{I}-h \mathcal{A} B(t))^{-1}\right\|_{0 \rightarrow 0} \leq\left\|\left(\mathcal{I}-h \mathcal{A} B_{0}(t)\right)^{-1}\right\|_{0 \rightarrow 0} \sum_{k=0}^{+\infty}\|\Delta(h)\|_{0 \rightarrow 0}^{k} \leq 2 K_{1} .
$$

Let $\bar{h}>0$ be the threshold given by Lemma 2.3. For $t \in J$ and $0<h<\bar{h}$ with $t+h \in J$, we set

$$
R(t, h)=(\mathcal{I}-h \mathcal{A} B(t))^{-1}, \quad R_{0}(t, h)=\left(\mathcal{I}-h \mathcal{A} B_{0}(t)\right)^{-1} .
$$

In this way, the discrete operator associated with the Runge-Kutta method in (5) is well defined for $0<h<\bar{h}$ and is given by

$$
r(t+h, t)=I+h \mathbf{b}^{T} B(t) R(t, h) \mathbf{e} .
$$

Furthermore, for $s, t \in J$ and $0<h<\bar{h}$ with $s+h, t+h \in J$, we set

$$
\delta(t, s, h)=B(t) R(t, h)-B_{0}(s) R_{0}(s, h) .
$$

(Several useful estimates for these operators are collected in the next lemma.)

In the rest of the paper $\bar{h}_{1}>0$ denotes the the minimum of the thresholds given in Lemmas 2.2 and 2.3 and of $L^{-1 / \alpha}$.

Lemma 2.4. There exists $K>0$, such that, for $s, t \in J, s \leq t$, and $0<h<\bar{h}_{1}$ with $t+h \in J$, the following estimates hold:

$$
\begin{aligned}
& \|R(t, h)\|_{\mu \rightarrow \mu}^{t \rightarrow t} \leq K, \quad\left\|R_{0}(t, h)\right\|_{\mu \rightarrow \mu}^{t \rightarrow t} \leq K, \quad 0 \leq \mu \leq 1, \\
& \|R(t, h)\|_{0 \rightarrow 1}^{t \rightarrow t} \leq K h^{-1}, \quad\left\|R_{0}(t, h)\right\|_{0 \rightarrow 1}^{t \rightarrow t} \leq K h^{-1}, \\
& \|\delta(t, s, h)\|_{\mu \rightarrow \nu}^{s \rightarrow t} \leq h^{\mu-\nu-1} K L \max \left\{(t-s)^{\alpha}, h^{\alpha}\right\}, \quad \mu, \nu \in[0,1], \\
& \|r(t+h, t)-\gamma\|_{0 \rightarrow 1}^{t \rightarrow t} \leq K h^{-1} .
\end{aligned}
$$

Proof. For $x \in X_{1}^{s}$ and $t \in J$, we set

$$
\|x\|_{1}^{* t}=\|x\|+\|B(t) x\|
$$

Because of $\mathrm{H} 2$ and the choice of $\bar{h}_{1}$, we have

$$
(1 / 2)\|x\|_{1}^{* t} \leq\|x\|_{1}^{t} \leq 2\|x\|_{1}^{* t}, \quad x \in X_{1}^{s}, \quad t \in J .
$$

By Lemma 2.3 we know that (21) holds for $\mu=0$. Then, by interpolation, only the case $\mu=1$ must be considered. Let $a>0$ be a bound for the norms of $\mathcal{A}, \mathcal{A}^{-1}$, $\mathbf{b}^{T}$ and $\mathbf{e}$ as operators in either the space $X_{0}$ or the space $X_{1}$, and let $K_{0}$ be the constant provided by Lemma 2.3. Notice that

$$
\begin{aligned}
B(t) R(t, h) & =B(t)(\mathcal{I}-h \mathcal{A} B(t))^{-1}=\mathcal{A}^{-1} \mathcal{A} B(t)(\mathcal{I}-h \mathcal{A} B(t))^{-1} \\
& =\mathcal{A}^{-1}(\mathcal{I}-h \mathcal{A} B(t))^{-1} \mathcal{A} B(t) .
\end{aligned}
$$


Hence, by (25),

$$
\begin{aligned}
\|R(t, h)\|_{1 \rightarrow 1}^{t \rightarrow t} & \leq 2\left(\|R(t, h)\|_{0 \rightarrow 0}^{t \rightarrow t}+\|B(t) R(t, h)\|_{1 \rightarrow 0}^{t \rightarrow t}\right) \\
& \leq 2\left(K_{0}+a^{2}\|R(t, h) B(t)\|_{1 \rightarrow 0}^{t \rightarrow t}\right) \\
& \leq 2\left(K_{0}+a^{2}\|R(t, h)\|_{0 \rightarrow 0}\|B(t)\|_{1 \rightarrow 0}^{t \rightarrow t}\right) \\
& \leq 2\left(K_{0}+2 a^{2} K_{0}\right) \\
& \leq 2\left(1+a^{2}\right) K_{0} ;
\end{aligned}
$$

thus, (21) holds for $R(t, h)$, with $K=4\left(1+a^{2}\right) K_{0}$.

Notice that we also have

$$
\begin{aligned}
B(t) R(t, h) & =\mathcal{A}^{-1} \mathcal{A} B(t)(\mathcal{I}-h \mathcal{A} B(t))^{-1} \\
& =h^{-1} \mathcal{A}^{-1}(R(t, h)-\mathcal{I}) .
\end{aligned}
$$

Hence, again by (25),

$$
\begin{aligned}
\|R(t, h)\|_{0 \rightarrow 1}^{t \rightarrow t} & \leq 2\left(\|R(t, h)\|_{0 \rightarrow 0}^{t \rightarrow t}+\|B(t) R(t, h)\|_{0 \rightarrow 0}^{t \rightarrow t}\right) \\
& \leq 2\left(K_{0}+h^{-1} a 2\left(K_{0}+1\right)\right),
\end{aligned}
$$

and (22) holds for $R(t, h)$.

The proofs of $(21)$ and $(22)$ for $R_{0}(t, h)$ are identical.

On the other hand, after some manipulation, we see that

$$
\begin{aligned}
\delta(t, s, h) & =h^{-1} \mathcal{A}^{-1}\left(h \mathcal{A} B(t) R(t, h)-h \mathcal{A} B_{0}(s) R_{0}(s, h)\right) \\
& =h^{-1} \mathcal{A}^{-1}\left(R(t, h)-R_{0}(s, h)\right) \\
& =\mathcal{A}^{-1} R(t, h) \mathcal{A}\left(B(t)-B_{0}(s)\right) R_{0}(s, h) .
\end{aligned}
$$

Therefore, for $\mu, \nu \in\{0,1\}$, we have

$$
\|\delta(t, s, h)\|_{\mu \rightarrow \nu}^{s \rightarrow t} \leq a^{2}\|R(t, h)\|_{0 \rightarrow \nu}^{t \rightarrow t}\left\|B(t)-B_{0}(s)\right\|_{1 \rightarrow 0}^{s \rightarrow t}\left\|R_{0}(s, h)\right\|_{\mu \rightarrow 1}^{s \rightarrow s} .
$$

By hypothesis $\mathrm{H} 2$ and (25), we also have

$$
\left\|B(t)-B_{0}(s)\right\|_{1 \rightarrow 0}^{s \rightarrow t} \leq 2 L(h+|t-s|)^{\alpha} \leq 8 L \max \left\{|t-s|^{\alpha}, h^{\alpha}\right\} .
$$

This estimate together with (21) and (22), in (26), yield (23), for $\mu=0,1$ and $\nu=0,1$. By interpolation, we obtain (23) for the remaining values $\mu, \nu \in(0,1)$.

Furthermore, we have

$$
\begin{aligned}
\|r(t+h, t)-\gamma\|_{0 \rightarrow 1}^{t \rightarrow t} & \leq\|r(t+h, t)-r(h A(t))\|_{0 \rightarrow 1}^{t \rightarrow t}+\|r(h A(t))-\gamma\|_{0 \rightarrow 1}^{t \rightarrow t} \\
& =h\left\|\mathbf{b}^{T} \delta(t+h, t, h) \mathbf{e}\right\|_{0 \rightarrow 1}^{t \rightarrow t}+\|r(h A(t))-\gamma\|_{0 \rightarrow 1}^{t \rightarrow t} \\
& =4 a^{2} L K h^{\alpha-1}+4 K e^{\omega_{0}} h^{-1} \\
& \leq 4\left(a^{2}+e^{\omega_{0} h}\right) K h^{-1},
\end{aligned}
$$

since $L h^{\alpha} \leq 1$.

\section{Proof of the main Result}

Proof of Theorem 1.1. Fix $0<h<\bar{h}_{1}$. We know that the stage equations are uniquely solvable for such values of $h$. Let $t_{j}, 0 \leq j \leq N$, be a sequence in $J$ with step size $h$. For $0 \leq j, n \leq N-1$, denote

$$
\begin{aligned}
\delta_{j, n} & =h^{-1}\left(r\left(t_{j+1}, t_{j}\right)-r\left(h A\left(t_{n}\right)\right)\right) \\
& =\mathbf{b}^{T} B\left(t_{j}\right)\left(\mathcal{I}-h \mathcal{A} B\left(t_{j}\right)\right)^{-1} \mathbf{e}-\mathbf{b}^{T} B_{0}\left(t_{n}\right)\left(\mathcal{I}-h \mathcal{A} B_{0}\left(t_{n}\right)\right)^{-1} \mathbf{e} \\
& =\mathbf{b}^{T} \delta\left(t_{j}, t_{n}, h\right) \mathbf{e}
\end{aligned}
$$


and, for $0 \leq n \leq m \leq N$ integers,

$$
F_{m, n}=\prod_{k=n}^{m-1} r\left(t_{k+1}, t_{k}\right)-\gamma^{m-n} \quad \text { and } \quad E_{m, n}^{j}=r^{m-n}\left(h A\left(t_{j}\right)\right)-\gamma^{m-n},
$$

where we take

$$
\prod_{k=n}^{n-1} r\left(t_{k+1}, t_{k}\right)=r^{0}\left(h A\left(t_{n}\right)\right)=\gamma^{0}=I
$$

We begin by proving (7). Let $0 \leq \mu<1$. Because of the well-known telescopic identity

$$
F_{m, n}=\sum_{j=n+1}^{m}\left(\prod_{l=j}^{m-1} r\left(t_{l+1}, t_{l}\right)\right)\left(r\left(t_{j}, t_{j-1}\right)-\gamma\right) \gamma^{j-n-1}
$$

and Lemmas 2.2 and 2.3, we conclude that $F_{m, n}$ is a bounded operator from $X$ to $X_{\mu}$. Let us point out that (27) is useless in order to establish bound (7), though the existence of the number $\left\|F_{m, n}\right\|_{0 \rightarrow \mu}^{t_{n} \rightarrow t_{m}}$ is required in the forthcoming argument. We also have the similar identity

$$
\begin{aligned}
F_{m, n}-E_{m, n}^{n} & =\sum_{j=n+1}^{m}\left(\prod_{l=j}^{m-1} r\left(t_{l+1}, t_{l}\right)\right)\left(r\left(t_{j}, t_{j-1}\right)-r\left(h A\left(t_{n}\right)\right)\right) r^{j-1-n}\left(h A\left(t_{n}\right)\right) \\
& =h \sum_{j=n+1}^{m}\left(F_{m, j}+\gamma^{m-j}\right) \delta_{j-1, n}\left(E_{j-1, n}^{n}+\gamma^{j-n-1}\right) .
\end{aligned}
$$

Hence, because of $(20)$,

$$
\begin{aligned}
\left\|F_{m, n}\right\|_{0 \rightarrow \mu}^{t_{n} \rightarrow t_{m}} & \leq\left\|E_{m, n}^{n}\right\|_{0 \rightarrow \mu}^{t_{n} \rightarrow t_{m}} \\
& +h \sum_{j=n+1}^{m}\left\|F_{m, j}\right\|_{0 \rightarrow \mu}^{t_{j} \rightarrow t_{m}}\left\|\delta_{j-1, n}\left(E_{j-1, n}^{n}+\gamma^{j-1-n}\right)\right\|_{0 \rightarrow 0}^{t_{n} \rightarrow t_{j}} \\
& +h \sum_{j=n+1}^{m} \kappa \gamma^{m-j}\left\|\delta_{j-1, n}\left(E_{j-1, n}^{n}+\gamma^{j-1-n}\right)\right\|_{0 \rightarrow \mu}^{t_{n} \rightarrow t_{j}},
\end{aligned}
$$

where $\kappa=\left(1+L T^{\alpha}\right)$.

On the one hand, by Lemma 2.2 , there exists $K_{1}$ such that $\left\|E_{j, n}^{n}\right\|_{0 \rightarrow \mu}^{t_{n} \rightarrow t_{n}} \leq$ $\left(K_{1} / 2\right) e^{\tilde{\omega}_{0}\left(t_{j}-t_{n}\right)}\left(t_{j}-t_{n}\right)^{-\mu}, 0 \leq j \leq N$. Moreover, by Lemmas 2.2 and 2.4, there exists a constant $K_{2}$ such that for $n+2 \leq j \leq m$ and either $\sigma=0$ or $\sigma=\mu$, we have

$$
\begin{aligned}
\left\|\delta_{j-1, n} E_{j-1, n}^{n}\right\|_{0 \rightarrow \sigma}^{t_{n} \rightarrow t_{j}} & \leq\left\|\delta_{j-1, n}\right\|_{1 \rightarrow \sigma}^{t_{n} \rightarrow t_{j}}\left\|E_{j-1, n}^{n}\right\|_{0 \rightarrow 1}^{t_{n} \rightarrow t_{n}} \\
& \leq 2 h^{-\sigma} L\left(K_{2} / 2\right) e^{\tilde{\omega}_{0}\left(t_{j-1}-t_{n}\right)}\left(t_{j}-t_{n}\right)^{\alpha}\left(t_{j-1}-t_{n}\right)^{-1} \\
& \leq 2 L K_{2} e^{\tilde{\omega}_{0}\left(t_{j-1}-t_{n}\right)} h^{-\sigma}\left(t_{j}-t_{n}\right)^{\alpha-1}
\end{aligned}
$$

and

$$
\left\|\delta_{j-1, n}\right\|_{0 \rightarrow \sigma}^{t_{n} \rightarrow t_{j}} \leq 2\left(K_{2} / 2\right) L h^{-1-\sigma}\left(t_{j-1}-t_{n}\right)^{\alpha} \leq 2 K_{2} L h^{-1-\sigma}\left(t_{j}-t_{n}\right)^{\alpha},
$$

since $\left(t_{j}-t_{n}\right) \leq 2\left(t_{j-1}-t_{n}\right)$ in the range $n+2 \leq j \leq m$. Furthermore, for $j=n+1$, by $(23)$, we have the analogous estimate

$$
\left\|\delta_{j-1, n}\right\|_{0 \rightarrow \sigma}^{t_{n} \rightarrow t_{j}}=\left\|\delta_{n, n}\right\|_{0 \rightarrow \sigma}^{t_{n} \rightarrow t_{n+1}} \leq 2 L\left(K_{2} / 2\right) h^{-1-\sigma} h^{\alpha} \leq 2 L K_{2} h^{-\sigma}\left(t_{j}-t_{n}\right)^{\alpha-1} .
$$


Thus, for $n+2 \leq j \leq m$, we deduce that

$$
\begin{aligned}
\| \delta_{j-1, n}\left(E_{j-1, n}^{n}\right. & \left.+\gamma^{j-1-n}\right) \|_{0 \rightarrow \sigma}^{t_{n} \rightarrow t_{j}} \\
& \leq\left\|\delta_{j-1, n} E_{j-1, n}^{n}\right\|_{0 \rightarrow \sigma}^{t_{n} \rightarrow t_{j}}+\left\|\delta_{j-1, n}\right\|_{0 \rightarrow \sigma}^{t_{n} \rightarrow t_{j}} \gamma^{j-1-n} \\
& \leq 2 L K_{2} e^{\tilde{\omega}_{0}\left(t_{j-1}-t_{n}\right)} h^{-\sigma}\left(\left(t_{j}-t_{n}\right)^{\alpha-1}+h^{-1}\left(t_{j}-t_{n}\right)^{\alpha} \gamma^{j-n-1}\right),
\end{aligned}
$$

and, for $j=n+1$, that

$$
\begin{aligned}
&\left\|\delta_{j-1, n}\left(E_{j-1, n}^{n}+\gamma^{j-1-n}\right)\right\|_{0 \rightarrow \sigma}^{t_{n} \rightarrow t_{j}} \\
& \leq 2 L K_{2} e^{\tilde{\omega}_{0}\left(t_{j-1}-t_{n}\right)} h^{-\sigma}\left(\left(t_{j}-t_{n}\right)^{\alpha-1}+h^{-1}\left(t_{j}-t_{n}\right)^{\alpha} \gamma^{j-n-1}\right) .
\end{aligned}
$$

Therefore, by using these estimates in (28), we get

$$
\begin{aligned}
\left\|F_{m, n}\right\|_{0 \rightarrow \mu}^{t_{n} \rightarrow t_{m}} \leq & \kappa K_{1} e^{\tilde{\omega}_{0}\left(t_{m}-t_{n}\right)}\left(t_{m}-t_{n}\right)^{-\mu} \\
& +2 L K_{2} h \sum_{j=n+1}^{m}\left\|F_{m, j}\right\|_{0 \rightarrow \mu}^{t_{j} \rightarrow t_{m}} e^{\tilde{\omega}_{0}\left(t_{j-1}-t_{n}\right)} \\
& \times\left(\left(t_{j}-t_{n}\right)^{\alpha-1}+h^{-1}\left(t_{j}-t_{n}\right)^{\alpha} \gamma^{j-n-1}\right) \\
& +2 \kappa L K_{2} e^{\tilde{\omega}_{0}\left(t_{m}-t_{n}\right)} h^{1-\mu} \\
& \times \sum_{j=n+1}^{m}\left(\gamma^{m-j}\left(t_{j}-t_{n}\right)^{\alpha-1}+h^{-1}\left(t_{j}-t_{n}\right)^{\alpha} \gamma^{m-n-1}\right) .
\end{aligned}
$$

For the proof of (7) we apply Lemma 2.1, considering that

$$
\xi_{j}=e^{-\tilde{\omega}_{0}\left(t_{m}-t_{m-j}\right)}\left\|F_{m, m-j}\right\|_{0 \rightarrow \mu}^{t_{m-j} \rightarrow t_{m}}, \quad 0 \leq j \leq m-n
$$

with $C_{1}=\kappa K_{1}, C_{2}=2 K_{2} L$ and $C_{3}=2 \kappa K_{2} L$. We take $\bar{h}$, the minimum of $\bar{h}_{1}$ and of the corresponding threshold given by Lemma 2.1. Notice that (20) is applied again, so that the presence of another factor $\kappa^{2}$ is needed in (7).

Assume now that $0<\nu \leq 1$. As before, identity (27) shows that $F_{m, n}$ is a bounded operator from $X_{\nu}$ onto $X_{1}$. Now we write

$$
F_{m, n}-E_{m, n}^{m}=h \sum_{j=n}^{m-1}\left(E_{m, j+1}^{m}+\gamma^{m-j-1}\right) \delta_{m, j}\left(F_{j, n}+\gamma^{j-n}\right) .
$$

Hence,

$$
\begin{aligned}
\left\|F_{m, n}\right\|_{\nu \rightarrow 1}^{t_{n} \rightarrow t_{m}} \leq & \left\|E_{m, n}^{m}\right\|_{\nu \rightarrow 1}^{t_{n} \rightarrow t_{m}} \\
& +h \sum_{j=n}^{m-1}\left\|\left(E_{m, j+1}^{m}+\gamma^{m-j+1}\right) \delta_{m, j}\right\|_{1 \rightarrow 1}^{t_{j} \rightarrow t_{m}}\left\|F_{j, n}\right\|_{\nu \rightarrow 1}^{t_{n} \rightarrow t_{j}} \\
& +h \sum_{j=n}^{m-1}\left\|\left(E_{m, j+1}^{m}+\gamma^{m-j+1}\right) \delta_{m, j}\right\|_{\nu \rightarrow 1}^{t_{j} \rightarrow t_{m}} \kappa \gamma^{j-n} .
\end{aligned}
$$


Let us take norms in (29). Arguing as before and using Lemmas 2.2 and 2.4, it is possible to prove that there exist constants $K_{1}$ and $K_{2}$ such that

$$
\begin{aligned}
& \left\|F_{m, n}\right\|_{\nu \rightarrow 1}^{t_{n} \rightarrow t_{m}} \leq \kappa K_{1} e^{\tilde{\omega}_{0}\left(t_{m}-t_{n}\right)}\left(t_{m}-t_{n}\right)^{\nu-1} \\
& \quad+2 L K_{2} h \sum_{j=n}^{m-1} e^{\tilde{\omega}_{0}\left(t_{m}-t_{j}\right)}\left(\left(t_{m}-t_{j}\right)^{\alpha-1}+h^{-1}\left(t_{m}-t_{j}\right)^{\alpha} \gamma^{m-j-1}\right)\left\|F_{j, n}\right\|_{\nu \rightarrow 1}^{t_{n} \rightarrow t_{j}} \\
& \quad+2 \kappa L K_{2} e^{\tilde{\omega}_{0}\left(t_{m}-t_{n}\right)} h^{\nu} \sum_{j=n}^{m-1}\left(\left(t_{m}-t_{j}\right)^{\alpha-1} \gamma^{j-n}+h^{-1}\left(t_{m}-t_{j}\right)^{\alpha} \gamma_{m-n}^{*}\right) .
\end{aligned}
$$

Now (8) is obtained by a direct application of Lemma 2.1, if we consider that $\xi_{j}=e^{-\tilde{\omega}_{0}\left(t_{n+j}-t_{n}\right)}\left\|F_{n+j, n}\right\|_{0 \rightarrow \mu}^{t_{n} \rightarrow t_{j+n}}, 0 \leq j \leq m-n, C_{1}=\kappa K_{1}, C_{2}=2 K_{2} L$, $C_{3}=2 \kappa K_{2} L$, and we take $\bar{h}$ the minimum of $\bar{h}_{1}$ and of the corresponding threshold given by Lemma 2.1 .

Finally, let us prove (9). Notice that now we cannot proceed as before, because the value $\eta=1$ is not covered by Lemma 2.1. If $N=1$, then we have directly

$$
\left\|F_{1,0}\right\|_{0 \rightarrow 1}^{t_{0} \rightarrow t_{1}} \leq\left\|E_{1,0}\right\|_{0 \rightarrow 1}^{t_{0} \rightarrow t_{1}}+\left\|\delta_{1,0}\right\|_{0 \rightarrow 1}^{t_{0} \rightarrow t_{1}} \leq 2 K_{2} h^{-1}+2 L K_{1} h^{-1},
$$

for some constants $K_{1}$ and $K_{2}$, as required. Let $N \geq 2$. We set $J=[N / 2]$ and write

$$
F_{N, 0}=F_{N, J+1} F_{J, 0}+\gamma^{N-J} F_{J, 0}+\gamma^{J} F_{N, J+1},
$$

whence

$$
\begin{aligned}
\left\|F_{N, 0}\right\|_{0 \rightarrow 1}^{t_{0} \rightarrow t_{N}} \leq \| & \left\|F_{N, J+1}\right\|_{0 \rightarrow 1 / 2}^{t_{J} \rightarrow t_{N}}\left\|F_{J, 0}\right\|_{1 / 2 \rightarrow 1}^{t_{0} \rightarrow t_{J}} \\
& \quad+\kappa \gamma^{N-J}\left\|F_{J, 0}\right\|_{0 \rightarrow 1}^{t_{0} \rightarrow t_{J}}+\kappa \gamma^{J}\left\|F_{N, J+1}\right\|_{0 \rightarrow 1}^{t_{J+1} \rightarrow t_{N}} .
\end{aligned}
$$

The first term on the right side of (30) is estimated by means of (7) and (8). Now let us estimate the central term $\gamma^{N-J}\left\|F_{J, 0}\right\|_{0 \rightarrow 1}^{t_{0} \rightarrow t_{J}}$. By (8) with $\nu=1$, we can bound

$$
\left\|\prod_{l=j}^{m-1} r\left(t_{l+1}, t_{l}\right)\right\|_{1 \rightarrow 1}^{t_{j} \rightarrow t_{m}} \leq \kappa \gamma^{m-j}+\left\|F_{m, j}\right\|_{1 \rightarrow 1}^{t_{j} \rightarrow t_{m}} \leq \kappa+\left\|F_{m, j}\right\|_{1 \rightarrow 1}^{t_{j} \rightarrow t_{m}} .
$$

Therefore, by taking norms in identity (27) with $m=J$ and $n=0$ and applying (24) we conclude that

$$
\begin{aligned}
& \gamma^{N-J}\left\|F_{J, 0}\right\|_{0 \rightarrow 1}^{t_{0} \rightarrow t_{J}} \\
& \quad \leq \gamma^{N-J} \sum_{j=1}^{J}\left\|\prod_{l=j}^{J-1} r\left(t_{l+1}, t_{l}\right)\right\|_{1 \rightarrow 1}^{t_{j-1} \rightarrow t_{J}}\left\|r\left(t_{j}, t_{j-1}\right)-\gamma\right\|_{0 \rightarrow 1}^{t_{j-1} \rightarrow t_{j-1}} \gamma^{j-1} \\
& \quad \leq \kappa \gamma^{N-J} \sum_{j=1}^{J} 2\left(\kappa+\left\|F_{J, j}\right\|_{1 \rightarrow 1}^{t_{j} \rightarrow t_{J}}\right) K h^{-1} \gamma^{j-1} .
\end{aligned}
$$

The final term is estimated in a similar way. These estimates together in (30) yield (9) because $\left\|F_{J, j}\right\|_{1 \rightarrow 1}^{t_{j} \rightarrow t_{J}}$ is bounded.

Remark. We can also prove the first part of Theorem 1.1 by constructing the discrete fundamental solution in a similar way to the continuous case. In fact, this is 
the idea used for the backward Euler method in [19]. For instance, when $\gamma=0$, it turns out that it is possible to obtain the representation

$$
F_{m, n}=r\left(h A\left(t_{n}\right)\right)^{m-n}+h \sum_{j=n}^{m} r\left(h A\left(t_{n}\right)\right)^{m-j} \Delta_{j, n}, \quad 0 \leq n \leq m \leq N,
$$

where $\Delta_{l, n}: X \rightarrow X$ are the linear and bounded operators defined by the recurrence

$$
\begin{aligned}
\Delta_{l-1, n}= & \delta_{l-1, n} r^{l-n-2}\left(h A\left(t_{n}\right)\right) \\
& +h \sum_{j=n}^{l-2} \delta_{l-1, j} r^{l-j-2}\left(h A\left(t_{j}\right)\right) \Delta_{j, n}, \quad 0 \leq n \leq l \leq N,
\end{aligned}
$$

starting from $\Delta_{n, n}=0$. These operators may be estimated by using Lemma 2.1.

\section{ACKNOWLEDGMENTS}

The first author has been supported by grant "Universidad de Valladolid". The second author has been supported by grant DGICYT PB-0254. The authors are also thankful to the anonymous referee for several valuable comments.

\section{REFERENCES}

[1] P. Acquistapace, Abstract linear nonautonomous parabolic equations: A survey, in Differential Equations in Banach Spaces, Proceedings 1991, Ed. G. Dore, A. Favini, E. Obrecht and A. Venni, Lectures Notes in Pure and Applied Mathematics 148, Dekker, New York, (1993), pp. 1-19. MR 94f:34113

[2] H. Amann, Parabolic evolution equations in interpolation and extrapolation spaces, J. Funct. Anal., 78 (1988), pp. 233-270. MR 90e: 47032

[3] H. Amann, Parabolic evolution equations and nonlinear boundary conditions, J. Diff. Eq., 72 (1988), pp. 201-269. MR 89e:35066

[4] N. Yu. BAKaev, Some problems of well-posedness of difference schemes on non-uniform grids, Comput. Math. Phys., 33 (1993), pp. 511-524. MR 94g:65085

[5] J. Bergh and J. Löfström, Interpolation Spaces. An Introduction, Springer-Verlag, Berlin, 1976. MR 58:2349

[6] P. Brenner and V. Thomée, On rational approximations of semigroups, SIAM J. Numer. Anal., 16 (1979), pp. 683-694. MR 80j:47052

[7] M. Crouzeix, S. Larsson, S. Piskarev and V. Thomée, The stability of rational approximations of analytic semigroups, BIT, 33 (1993), pp. 74-84. MR 96f:65069

[8] H.O. Fattorini, The Cauchy Problem, Addison-Wesley, Reading MA, 1983. MR 84g:34003

[9] H. Fujita and T. Suzuki, Evolution Problems, Handbook of numerical analysis, Ed. P. G. Ciarlet and J. L. Lions, North-Holland, Amsterdam, 1991. CMP 91:14

[10] C. González and C. Palencia, Stability of time-stepping methods for time-dependent parabolic problems, SIAM J. Numer. Anal., 35 (1998), pp. 973-989.

[11] C. González and C. Palencia, Stability of Runge-Kutta methods for abstract quasilinear parabolic problems in Banach spaces, submitted to Math. Comp.

[12] E. Hairer and G. Wanner, Solving Ordinary Differential Equations II. Stiff Problems, Springer-Verlag, Berlin (1988).

[13] S. G. Krein, Linear Differential Equations in Banach Space, Amer. Math. Soc., Providence, 1971. MR 49:7548

[14] S. Larsson, V. Thomée And L. B. Wahlbin, Numerical solution of parabolic integrodifferential equations by the discontinuous Galerkin method, Preprint 1995:5, Department of Mathematics, Chalmers University of Technology and Göteborg University, Göteborg, 1995.

[15] Ch. Lubich And A. Ostermann, Runge-Kutta approximation of quasilinear parabolic equations, Math. Comp., 64 (1995), pp. 601-627. MR 95g:65122

[16] C. PAlencia, A stability result for sectorial operators in Banach spaces, SIAM J. Numer. Anal., 30 (1993), pp. 1373-1384. MR 94j:65109 
[17] C. Palencia, Stability of rational multistep approximations of holomorphic semigroups, Math. Comp., 64 (1995), pp. 591-599. MR 95g:65079

[18] A. PAZY, Semigroups of Linear Operators and Application to Partial Differential Equations, Springer-Verlag, New York, (1983). MR 85g:47061

[19] P. E. Sobolevskit, Equations of parabolic type in Banach space, Amer. Math. Soc. Transl., 49 (1966), pp. 1-62.

[20] P. E. Sobolevskit, The theory of semigroups and stability of difference schemes, Culc. c. of Sibirien AN USSR. School on theory of operators in functional spaces (25-31 August), Novosibirsk, Preprint, (1975), pp. 1-38.

[21] H. Tanabe, Equations of Evolution, Pitman, London, 1979. MR 89g:47032

[22] H. Triebel, Interpolation Theory, Function Spaces, Differential Operators, North-Holland, Amsterdam, 1978. MR 80i:46032b

Departamento de Matemática Aplicada y Computación, Universidad de Valladolid, VAlladolid, Spain

E-mail address: cesareo@mac.cie.uva.es

E-mail address: palencia@cpd.uva.es 